\title{
Invasiveness of plant pathogens depends on the spatial scale of host distribution
}

Article

Accepted Version

Mikaberidze, A., Mundt, C. C. and Bonhoeffer, S. (2016)

Invasiveness of plant pathogens depends on the spatial scale of host distribution. Ecological Applications, 26 (4). pp. 12381248. ISSN 0051-0761 doi: https://doi.org/10.1890/15-0807 Available at https://centaur.reading.ac.uk/86101/

It is advisable to refer to the publisher's version if you intend to cite from the work. See Guidance on citing.

To link to this article DOI: http://dx.doi.org/10.1890/15-0807

Publisher: Ecological Society of America

All outputs in CentAUR are protected by Intellectual Property Rights law, including copyright law. Copyright and IPR is retained by the creators or other copyright holders. Terms and conditions for use of this material are defined in the End User Agreement.

\section{www.reading.ac.uk/centaur}

\section{CentAUR}

Central Archive at the University of Reading

Reading's research outputs online 


\title{
Invasiveness of plant pathogens depends on the spatial scale of host distribution
}

\author{
Alexey Mikaberidze ${ }^{*, 1}$, \\ Christopher C. Mundt ${ }^{2}$, \\ Sebastian Bonhoeffer ${ }^{1}$
}

*alexey.mikaberidze@env.ethz.ch, Institute of Integrative Biology, ETH Zurich, CHN H 75.1, Universitaetstrasse 16, 8092, Zurich, phone: +41 446322602

${ }^{1}$ Institute of Integrative Biology, ETH Zurich

${ }^{2}$ Department of Botany and Plant Pathology, Oregon State University 


\begin{abstract}
Plant diseases often cause serious yield losses in agriculture. A pathogen's invasiveness can be quantified by the basic reproductive number, $R_{0}$. Since pathogen transmission between host plants depends on the spatial separation between them, $R_{0}$ is strongly influenced by the spatial scale of the host distribution.

We present a proof of principle of a novel approach to estimate the basic reproductive number, $R_{0}$, of plant pathogens as a function of the size of a field planted with crops and its aspect ratio. This general approach is based on a spatially-explicit population dynamical model. The basic reproductive number was found to increase with the field size at small field sizes and to saturate to a constant value at large field sizes. It reaches a maximum in square fields and decreases as the field becomes elongated. This pattern appears to be quite general: it holds for dispersal kernels that decrease exponentially or faster as well as for fat-tailed dispersal kernels that decrease slower than exponential (i.e. power-law kernels).

We used this approach to estimate $R_{0}$ in wheat stripe rust (an important disease caused by Puccinia striiformis), where we inferred both the transmission rates and the dispersal kernels from the measurements of disease gradients. For the two largest datasets, we estimated $R_{0}$ of $P$. striiformis in the limit of large fields to be of the order of 30 . We found that the spatial extent over which $R_{0}$ changes strongly is quite fine-scaled (about $30 \mathrm{~m}$ of the linear extension of the field). Our results indicate that in order to optimize the spatial scale of deployment of fungicides or host resistances, the adjustments should be made at a fine spatial scale. We also demonstrated how the knowledge of the spatial dependence of $R_{0}$ can improve recommendations with regard to fungicide treatment.
\end{abstract}

Keywords: basic reproductive number, disease control, disease gradient, dispersal, epidemiology, host-pathogen interaction, mathematical model, plant disease, population dynamics, spatial scales 


\section{Introduction}

When plant pathogens succeed in infecting their hosts, they colonize the host tissue and deprive hosts of resources and energy. This often leads to serious yield losses in agriculture (Strange and Scott, 2005). Disease-resistant crop varieties and chemicals (fungicides or antibiotics) are widely used to control infectious diseases of plants. But both of these control measures are highly vulnerable to pathogen adaptation: pathogens evolve to overcome host resistances and to become insensitive to fungicides (McDonald and Linde, 2002). In order to devise effective and durable strategies of disease control (Mundt, 2014), a thorough understanding of basic epidemiological properties of plant pathogens with the help of appropriate mathematical models is necessary. The spread of infectious diseases depends on the contact structure, a network in which each host is a node and has a number of weighted, directional links to other hosts. The strength of each link represents the probability of transmission from one host to another. In infectious diseases of humans and animals contact structures are determined by networks of social contacts. Plant pathogens spread over global scales of countries and continents by natural means and through networks of trade and exchange (Brown and Hovmoller, 2002; Shaw and Pautasso, 2014). However, at a local scale of a single field of crop plants or several adjacent fields, plant pathogens spread primarily through passive dispersal of infectious propagules through air, water or soil between immobile plants. Insect pests may disperse both actively and passively between hosts plants (Mazzi and Dorn, 2012). In both of these cases, the probability of transmission between hosts depends on the geographical distance between them. Hence, the contact structure is determined by the spatial scales of pathogen dispersal and the spatial scales of the host population.

Full information on the contact structure is difficult to obtain and to analyze. Several global measures are used to characterize networks of contacts, such as the average degree, i. e. the average 
number of links per host. Yet, a better measure that characterizes the disease spread is its basic reproductive number, $R_{0}$, defined intuitively as "the average number of secondary cases of infection generated by one primary case in a susceptible host population" (Anderson and May, 1986). Mathematically, it is given by the dominant eigenvalue of the next generation operator (Heesterbeek, 2002). Hence, the basic reproductive number is a quantity with a clear biological meaning that characterizes reproductive fitness of the pathogen. In deterministic models, it determines the invasion threshold: if $R_{0}>1$ the disease will spread in the population, otherwise at $R_{0}<1$ the pathogen will eventually die out. Therefore, $R_{0}$ can be used to estimate the critical proportion of the host population that needs to be immunized (i. e. vaccinated) in order to eradicate the disease (Anderson and May., 1991). Also, $R_{0}$ often allows one to estimate the final (equilibrium) disease level.

Much attention has been devoted to estimation of $R_{0}$ for infectious diseases of humans and animals (Anderson and May., 1991; Fraser et al., 2009; Hampson et al., 2009). Several studies discuss $R_{0}$ in the context of infectious diseases of plants (Gubbins et al., 2000; Park et al., 2001; Parnell et al., 2005; van den Bosch et al., 2008; van den Berg et al., 2011), and in (Montarry et al., 2010) was estimated for potato late blight. But only one study provided estimates for wheat stripe rust (Segarra et al., 2001) based on measurements of the apparent infection rate $r$ (the rate of growth of the disease proportion over time, assuming logistic growth (Vanderplank, 1963)). Another approach is to estimate $R_{0}$ by fitting the solution of a population dynamics model of disease spread to an empirical disease progress curve (i. e. the plot of the proportion of disease over time). However, this appears to be difficult, because we expect $R_{0}$ to depend on the spatial scales of the host population. In an agricultural setting, crop plants are usually arranged in nearly rectangular fields. Each field is characterized by its area $S$ and aspect ratio $\alpha$. Hence, $R_{0}$ should depend on $S$ and $\alpha$, provided that the planting density is fixed. Given the wide variation in field sizes and shapes across individual 
fields and growing regions, countries and continents, a useful estimate for $R_{0}$ should also capture the dependence on the field size and shape. But measuring disease progress curves in many fields with different sizes and shapes requires enormous efforts and resources.

In this study we propose a novel way to estimate the basic reproductive number $R_{0}$ as a function of field size and shape. This approach uses a spatially explicit population dynamics model formulated as a system of integro-differential equations. In this approach, estimation of $R_{0}$ requires knowledge of a dispersal kernel, a function that describes dispersal properties of the pathogen. In general, estimation of dispersal kernels is a highly non-trivial problem, as often only limited spatially-resolved disease data is available. Here, we estimate dispersal kernels using disease gradient measurements in which the amount of disease is characterized as a function of the distance from a localized source of initial inoculum. These measurements are only possible for some plant disease systems, while in other cases only limited disease data can be obtained. In these cases, more elaborate statistical methods are necessary to estimate dispersal kernels (Filipe et al., 2012;

Gibson et al., 2006).

To provide a proof of principle for this method of estimating $R_{0}$, we applied it to wheat stripe rust (an important pathogen of wheat caused by Puccinia striiformis (Wellings, 2011)), since disease gradients for this pathogen were thoroughly measured over large distances (Sackett and Mundt, 2005a; Cowger et al., 2005). Using these data, we estimated $R_{0}$ as a function of the field size and shape. From this dependence we determined the ranges of field sizes and shapes over which $R_{0}$ exhibits a considerable change. The advantage of this approach is that, by measuring the disease gradient over a large enough distance in a single experiment, one captures the information on the dependence of $R_{0}$ on the field size and aspect ratio. In this way, more useful information can be extracted from disease gradient data than thought previously. 
96

\section{Methods}

We assume that the hosts are continuously distributed across the rectangular field with the dimensions $d_{x}$ and $d_{y}$. The field area is $S=d_{x} d_{y}$ and its aspect ratio is $\alpha=d_{x} / d_{y}$, so that $\alpha$ close to zero refers to long, narrow fields, while $\alpha=1$ represents a square field. We trace the densities of healthy hosts $H(x, y, t)$, infected hosts $I(x, y, t)$ and removed hosts $R(x, y, t)$ in space and time using the system of integro-differential equations

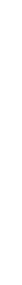

$$
\begin{aligned}
& \frac{\partial H(x, y, t)}{\partial t}=r_{H} H(x, y, t)[1-H(x, y, t) / K]-\beta \lambda(x, y) H(x, y, t) \\
& \frac{\partial I(x, y, t)}{\partial t}=\beta \lambda(x, y) H(x, y, t)-\mu I(x, y, t) . \\
& \frac{\partial R(x, y, t)}{\partial t}=\mu I(x, y, t) .
\end{aligned}
$$

Here, the force of infection $\lambda(x, y)$ at a location $x, y$ is determined by integrating over all possible sources of infection:

In obtaining Eqs. (1)-(3) we assumed that the characteristic time scale of spore dispersal is much shorter than the characteristic time scales associated with other stages of the pathogen life cycle and, hence, the density of spores is proportional to the density of the infectious host tissue (see Appendix A.4 in Supporting Information for more details). We also neglected the latent compartment consisting of hosts that are infected but not yet infectious, in spite of the fact that average duration of latent infection was estimated to be around 10 days in wheat stripe rust (van den Bosch et al., 1988; Sache and Vallavieille-Pope, 1993). This simplification is justified because here we focus on determining $R_{0}$, the basic reproductive number, at the starting phase of 
the epidemic. This phase corresponds to the beginning of the growing season of wheat, when the senescence of leaves (natural death of hosts) is negligibly small. Under these conditions, latently infected hosts are likely to survive and be transformed into infectious hosts. Hence, the basic reproductive is not affected by latency. At the same time, latency of stripe rust played an important role in distinguishing the primary disease gradients from subsequent pathogen generations when collecting the data (Sackett and Mundt, 2005a) that we analyze here.

The quantities $H(x, y, t), I(x, y, t)$ and $R(x, y, t)$ represent the areas of the corresponding host tissue per unit land area. The host tissue could be leaves, stems or grain, depending on the specific host-pathogen interaction. Healthy hosts $H(x, y, t)$ grow logistically with the rate $r_{H}$ and the "carrying capacity" $K$, which may imply limited space or nutrients. We consider a single growing season and neglect leaf senescence. Furthermore, healthy hosts may be infected by the pathogen and transformed into infected hosts with the rate $\beta \lambda(x, y)$. The transmission rate $\beta$ is a compound parameter given by the product of the sporulation rate of the infected tissue and the probability that a spore causes new infection. Infected host tissue loses its infectivity at a rate $\mu$, where $\mu^{-1}$ is the average infectious period. In this way, infected hosts $I(x, y, t)$ are continuously transformed into removed hosts $R(x, y, t)$. Here, $R(x, y, t)$ quantifies the amount of host tissue that was previously infected, but subsequently lost its ability to produce infectious spores and cannot be re-infected. To complete the initial-value problem, the initial conditions for the system of Eqs. (1)-(3) can be defined as, $H(x, y, t=0)=H_{0}(x, y), I(x, y, t=0)=I_{0}(x, y), R(x, y, t=0)=R_{0}(x, y)$. Most of the results of this study were obtained from the solution of the eigenvalue problem [Eq. (6) below] that does not require initial conditions. The results in Sec. 3.3 were obtained using an approximate spatially uniform model. Accordingly, in Sec. 3.3 we used spatially uniform initial conditions: $H(x, y, t=0)=H_{0}, I(x, y, t=0)=I_{0}, R(x, y, t=0)=0$. The border condition requires $H(x, y, t)=0$ for all $x, y$ that lie outside the field. An approximate version of the model 
Eqs. (1)-(3), in which the host densities were assumed to be homogeneous in space, was used in several previous studies of plant disease epidemics (Hall et al., 2007; van den Bosch and Gilligan, 2008; Mikaberidze et al., 2014b).

The integral in Eq. (4) is weighted using $\kappa(x, y, u, v)$, the dispersal kernel (or contact distribution (Mollison, 1977)) that characterizes dispersal properties of the pathogen. The dispersal properties as well as the environmental conditions are assumed to be the same along the field. Moreover, dispersal is assumed to be isotropic, meaning that a spore has the same probability to move in any direction along the two-dimensional field. The latter assumption can be problematic when strong winds prevail in a certain direction and may be the cause of discrepancy with the empirical findings. In this case, the dispersal kernel is only determined by the distance

$$
r=\sqrt{(x-u)^{2}+(y-v)^{2}}
$$

between the source and the target of infection, i. e. $\kappa(x, y, u, v)=\kappa(r)$. For aerially dispersed plant diseases, $\kappa(r)$ is defined as a probability density function for an infectious spore to land at a distance $r$ from its source (Nathan et al., 2012).

In order to determine the basic reproductive number, $R_{0}$, we perform the linear stability analysis of the disease-free equilibrium $H(x, y, t)=K, I(x, y, t)=0, R(x, y, t)=0$ of the system Eqs. (1)-(2). Essentially, we examine whether small deviations from the disease-free equilibrium grow or die out. This leads to the eigenvalue problem for the Fredholm equation of the second kind (see Appendix A.1 for the derivation)

$$
R_{0 \infty} \int_{0}^{d_{x}} d u \int_{0}^{d_{y}} d v \kappa(r) w(u, v)=\sigma w(x, y)
$$

where $R_{0 \infty}=\beta K / \mu$ is the basic reproductive number in the limit of a very large field. Definition 
of $R_{0 \infty}$ here, requires that $\mu>0$. This holds for wheat stripe rust, where the infectious period was estimated to be $\mu^{-1} \approx 30$ days (Sache and Vallavieille-Pope, 1993). Also, this infectious period is shorter than the duration of the growing season of wheat. By solving the eigenvalue problem Eq. (6), we can find the eigenvalues $\sigma_{i}$ and eigenfunctions $w_{i}(x, y)$ that satisfy the Eq. (6). The dominant eigenvalue $\sigma_{d}$ determines the basic reproductive number, i. e. $R_{0}=\sigma_{d}$.

An approximate expression for the basic reproductive number for the model Eqs. (1)-(2) can be found by applying its intuitive definition (Anderson and May, 1986) and averaging over the spatial coordinates. This leads to the expression:

$$
R_{0 \mathrm{c}}\left(x_{0}, y_{0}\right)=\frac{\beta K}{\mu} \int_{0}^{d_{x}} d x \int_{0}^{d_{y}} d y \kappa\left(x, y, x_{0}, y_{0}\right)
$$

where we set $H(x, y, t=0)=K, I(x, y, t=0)=I_{\text {tot0 }} \delta\left(x-x_{0}\right) \delta\left(y-y_{0}\right), R(x, y, t=0)=0$. Here, the approximate basic reproductive number depends on the position $x_{0}, y_{0}$ of the initial inoculum, in contrast to the exact basic reproductive number determined from Eq. (6), which is independent on the position of the initial inoculum. The approximate basic reproductive number in Eq. (7) does not yield the invasion threshold at $R_{0 \mathrm{c}}\left(x_{0}, y_{0}\right)=1$ (Diekmann et al., 1990). However it may serve as a useful approximate expression, since the calculation according to Eq. (7) is often much simpler than the solution of the eigenvalue problem Eq. (A.3). We found (Appendix A.2) that the approximate expression for the basic reproductive number Eq. (7) generally underestimates the actual $R_{0}$, because it neglects the contribution of the subsequent generations of infection. But it holds well in the two limiting cases: at small field sizes (i.e. when $d \ll a$ ) and at large field sizes (i. e. when $d \gg a$ ).

$R_{0}$ is computed by numerically solving the eigenvalue problem in Eq. (6) for different values of the field dimensions $d_{x}$ and $d_{y}$ that characterize the field size and shape. Before performing this 
calculation, we estimated the dispersal kernel $\kappa(r)$ and the compound parameter $R_{0 \infty}$ from experimental data (Sackett and Mundt, 2005a; Cowger et al., 2005) [see Appendix A.3 for the details of the estimation procedure].

In these experiments, winter wheat cultivar Jacmar was planted in three replicate plots measuring $6.1 \mathrm{~m}$ wide by 128 to $171 \mathrm{~m}$ long, oriented parallel to the dominant wind direction (see Fig. 1 in (Sackett and Mundt, 2005a)). Small areas of experimental plots (foci) were artificially inoculated by pathogen spores ( $\left(0^{\text {th }}\right.$ generation). These spores give rise to lesions in the focus (first generation) that further produce spores, which are dispersed through the air. This results in infection outside of the focus, producing the second generation of pathogen lesions. The corresponding disease severity (the proportion of the leaf area infected) is measured as a function of the distance $r$ from the focus. We are confident that the observed disease patterns result primarily from the artificial inoculum in the focus, rather than natural infection, because stripe rust levels were overall very light in Oregon in 2002 (Long, 2003). Further, disease levels declined consistently with distance and often reached zero at the farther distances from the inoculum source, which would not be expected if there was significant inoculum from outside of the plots.

When considering fungicide treatment (Sec. 3.3), we take into account the effect of the fungicide on the transmission rate of the pathogen using the expression

$$
\beta(D)=\beta_{0}[1-\varepsilon(D)],
$$

where the fungicide efficacy, $\varepsilon(D)$, is given by the Hill's function

$$
\varepsilon(D)=\varepsilon_{\mathrm{m}} \frac{D}{D+D_{50}}
$$

and describes the proportion by which the transmission rate is reduced by the fungicide. In Eq. (9), 
$D$ is the fungicide dose, $\varepsilon_{\mathrm{m}}$ is the maximum fungicide effect at high doses, and $D_{50}$ is the dose at which half of the maximum effect is achieved.

\section{Results}

\subsection{Dependence of the basic reproductive number on the field size}

The basic reproductive number, $R_{0}$, is shown in Fig. 1 as a function of the linear extension $d$ of a square field for three different dispersal kernels (Gaussian, exponential and modified power-law). These three functional forms are often used to describe dispersal gradients in plant diseases (Fitt et al., 1987; Frantzen and Bosch, 2000; Sackett and Mundt, 2005a), but also in other taxonomic groups, for example, in pollen, seeds, seedlings, beetles, moths and butterflies (Nathan et al., 2012). These three functions represent the three classes of dispersal kernels: "thin-tailed" (Gaussian) that decrease faster than exponential, exponential, and "fat-tailed" that decrease slower than exponential (power-law). "Thin-tailed" and exponential kernels give rise to travelling epidemic waves with a constant velocity, while the "fat-tailed" kernels result in accelerating epidemic waves (Mollison, 1977; Medlock and Kot, 2003; Cowger et al., 2005; Sackett and Mundt, 2005b).

For all the three types of dispersal kernels that we considered, the basic reproductive number first increases as a function of the field size $d$ and then, eventually, saturates to a constant value (Fig. 1).

Thus, we find that the qualitative dependence of $R_{0}$, a more basic epididemiological parameter than the epidemic velocity, on the field size is quite robust with respect to the functional form of the dispersal kernel. In particular, it is not affected much by the nature of the tails of the dispersal kernel. Moreover, we expect this behaviour to hold for any dispersal kernel, as long as it a monotonically decreasing function of the distance $r$.

The initial growth of $R_{0}$ versus $d$ follows a quadratic function (see Eq. (A.10)). It occurs because in 
this range, the field size is much smaller than the dispersal radius $a$ (a characteristic length scale of pathogen dispersal), i.e. $d \ll a$. Therefore, by making the field larger, more spores will land within the field and lead to new infections. In other words, in this range the field size is the limiting factor for the pathogen fitness. On the contrary, when the field size is much larger than the dispersal radius, i. e. $d \gg a$, the basic reproductive number becomes independent of $d$. Here, pathogen does not become fitter on a larger field, because its fitness is now limited by the range of dispersal and not by the size of the field.

While the three curves in Fig. 1 exhibit a universal qualitative behaviour, they differ in the rate at which the saturation occurs at large field sizes. The Gaussian dispersal kernel decreases faster with the distance $r$ than the exponential dispersal kernel. As a result, $R_{0}$ grows and saturates as a function of the field size $d$ faster for the Gaussian than for the exponential. The result for the power-law dispersal kernel is difficult to compare with the results for other kernels, since the power law lacks a meaningful characteristic length scale. Asymptotically, at large field sizes $R_{0}$ approaches the constant value slower in the case of the power-law dispersal kernel than for the other two kernels. However, at small field sizes, $R_{0}$ as a function of $d$ may grow faster or slower for the power-law kernel as compared to the other two kernels, depending on the values of the parameters $r_{0}$ and $b$. In Fig. 1, we present an example when the $R_{0}$ for the power law first grows faster than that for the Gaussian or exponential dispersal kernels, but subsequently its growth slows down and becomes slower than for the Gaussian and exponential (as expected from the asymptotic behavior of the corresponding dispersal kernels). 


\subsection{Case study: dependence of the basic reproductive number on the} field size and shape for wheat stripe rust

We infer the dependence of the basic reproductive number, $R_{0}$, on the field size and shape from the detailed measurements of primary disease gradients of wheat stripe rust (Sackett and Mundt, 2005a; Cowger et al., 2005). The outcome of this measurement is shown in Fig. 2 for the two largest datasets (Hermiston 2002 and Madras 2002, downwind) obtained in this experiment. These two datasets were chosen because they contained measurements over large enough distances that allowed us to obtain sound fits. Disease severity strongly depends on the distance $r$ : the value is largest closer to the focus and decreases monotonically with $r$. The data can be fitted well by the modified power-law function (solid curve in Fig. 2)

$$
\kappa_{\mathrm{PL} 2}(r)=\kappa_{0}\left(r_{0}^{2}+r^{2}\right)^{-b / 2}
$$

In contrast, exponential and Gaussian functions provide poor fits (dashed and dotted curves in Fig. 2). (For more details on fitting see Appendix A.3.1 and Fig. 6 in the Electronic Supplementary Materials).

Disease gradients, measured in this way, contain information on the three key processes in the pathogen life-cycle: spore production, aerial movement of spores, and infection of healthy host tissue. We assume that the rate of spore production and the probability to infect healthy host tissue, once the spore has landed on it, are homogeneous across the field, i. e. do not depend on the distance $r$ between the source and the target of infection [Eq. (5)]. Hence, the compound parameter $R_{0 \infty}=\beta K / \mu$ that characterizes these processes does not depend on the distance $r$. Therefore, the aerial movement of spores is the only process that depends on the distance $r$. Further, we assume that there is a large enough number of spores produced and the probability of infection is large 
enough such that the recorded disease severity is proportional to the spore concentration in the air. Under these assumptions, our estimate for the dispersal kernel $\kappa(r)$ is the modified power-law function [Eq. (10)] fitted to the disease gradient data and normalized as a probability density function (i.e. such that its integral over the whole two-dimensional space equals to unity [Appendix A.3.2]). We also estimated the parameter $R_{0 \infty}$ from the disease gradient data (see Appendix A.3.3) and obtained the value $R_{0 \infty}=35 \pm 3$ for the Hermiston 2002 downwind dataset; and the value $R_{0 \infty}=23 \pm 4$ for the Madras 2002 downwind dataset.

Using our estimates for the dispersal kernel, $\kappa(r)$, and the parameter $R_{0 \infty}$ we solved the eigenvalue problem Eq. (6) numerically for different field sizes and shapes. In this way, we obtained the dependence of the basic reproductive number $R_{0}$ on the field size (Fig. 3) and its aspect ratio (Fig. 4). In Fig. 3, $R_{0}$ first grows steeply versus the linear extension of a square field and saturates towards the asymptotic value $R_{0 \infty}$ for large fields. The basic reproductive number is about two times larger for the parameter values corresponding to Hermiston 2002 dataset, than for the case of Madras 2002 dataset. This difference stems from the difference in the asymptotic values $R_{0 \infty}$ and also from different shapes of the disease gradients (cf. panel (a) and (b) in Fig. 2). The asymptotic value, $R_{0 \infty}$, (indicated by the horizontal dashed line in Fig. 3), is approached faster in the case of Hermiston 2002 dataset (solid curve in Fig. 3), than for Madras 2002 dataset (dashed curve in Fig. 3). The reason for this is a different exponent of the power-law function that best fits the corresponding disease gradients ( $b=3.04$ for Hermiston 2002, Eq. (A.15), and $b=2.23$, Eq. (A.16)). The disease gradient in Madras 2002 decreases slower due a lower exponent. In Fig. $4, R_{0}$ exhibits a saturating growth as the field aspect ratio $\alpha$ is increased from 0.01 to 1. Hence, the square fields, with $\alpha=1$, are most conducive for the disease growth. The basic reproductuve number grows faster and saturates at larger values of $\alpha$ in smaller fields (cf. dotted, dashed, dash-dotted and solid curves in Fig. 4). 
A number of empirical studies have reported that, in agreement with our results, smaller plots resulted in lower disease levels in wheat stripe rust (Mundt et al., 1996), wheat brown rust (Puccinia recondita f. sp. tritici) (Bowen et al., 1984), potato late blight (Paysour and Fry, 1983) and Valdensia heterodoxa on Vaccinium myrtillus (Strengbom et al., 2006). However, in a more recent study in wheat stripe rust (Sackett and Mundt, 2009) that used considerably larger plot sizes, the plot size did not affect the epidemic velocity. Our estimation framework predicts moderate differences in the values of $R_{0}$ between larger square plots and smaller elongated plots used in experiments (Sackett and Mundt, 2009) (cf. the white and gray circles in both panels of Fig. 4). This is expected to result in higher epidemic velocities in larger plots compared to smaller plots, according to theoretical arguments (Keeling and Rohani, 2008). We suggest two possible explanations for this discrepancy. First, strong wind with a prevailing direction along the axis of the elongated plot was observed in the experimental setting (Sackett and Mundt, 2009), but in our model isotropic dispersal was assumed. The differences in $R_{0}$ between smaller elongated plot and a larger square plot that we predict using the model are possibly masked by the wind. This is because the wind may increase the pathogen's $R_{0}$ in the smaller elongated plot by preventing the spores to land outside the plot. Second, a moderate difference of 20-30\% that we predict for epidemic velocities may be difficult to detect given the level of experimental uncertainties.

\subsection{Effect of the plot size on the fungicide dose-response}

Control of stripe rust greatly relies on fungicides. Field experiments, in which disease severity is measured as a function of the fungicide dose, inform strategies of fungicide treatement. These experiments are typically performed on rather small fields, in the range of $20-60 \mathrm{~m}^{2}$. The outcomes are used to choose appropriate fungicide doses (Paveley et al., 1998).

How do the sizes of experimental plots affect the outcomes of dose-response measurements? What 
implications does it have for disease control? Figure 5 illustrates the effect of the plot size on the fungicide dose-response as predicted by the model. Severity of stripe rust [panel (a)] and the pathogen's basic reproductive number, $R_{0}$, [panel (b)] are shown versus the fungicide dose for two field sizes: $60 \mathrm{~m}^{2}$ (solid) and $90 \mathrm{~m}^{2}$ (dashed). We fitted the solid curve in Fig. 5 (a) to the dose-response data (Bounds et al., 2012) (filled triangles), while the other three curves in Fig. 5(a,b) were devised from it using theoretical considerations. In particular, we chose to use a non-spatial approximation to the full solution of the system of Eq. (1)-(3), which assumes homogeneous mixing and more specifically assumes that the disease severity changes over time according to a logistic function [Eq. (A.49) in Appendix A.5]. This choice is justified, because there is no availability of empirical data on spatial dynamics within individual field-plots in fungicide dose-response experiments. Nevertheless, the effect of the spatial extension is incorporated through the dependence of the basic reproductive number, $R_{0}$, on the field size that was determined above. We also assumed that the field size and the fungicide affect the basic reproductive number independently, hence their effects enter as multiplicative factors. (Please refer to Appendix A.5 for details).

Disease severity strongly depends on both the fungicide dose and the field size (cf. solid and dashed curves in Fig. 5(a)). $R_{0}$ also depends on both the fungicide dose and the field size (cf. solid and dashed curves in Fig. 5(b)), but the changes in $R_{0}$ are less pronounced than changes in disease severity. Hence, in this parameter regime of large $R_{0}$-values, moderate changes in $R_{0}$ may lead to considerable changes in disease severity.

What do these insights mean for disease control? First, achieving good disease control in a relatively small plot $\left(\lesssim 100 \mathrm{~m}^{2}\right)$ does not guarantee good control in larger fields. For example, in the $60 \mathrm{~m}^{2}$ plot, disease severity of $<5 \%$ was reached at a fungicide dose $D=0.25$ [solid curve in Fig. 5(a)]. But in a larger field of $90 \mathrm{~m}^{2}$ this dose was able to reduce the severity only down to about 
$30 \%$ [dashed curve in Fig. 5(a)]. Therefore, typical dose-response measurements that are performed in the range of field sizes of $30-60 \mathrm{~m}^{2}$ may strongly underestimate the disease severity in larger fields used by growers. As a result, recommendations with regard to fungicide dosage and other control options based on dose-response measurements in small fields may be unreliable. However, a considerable increase in sizes of experimental plots seems impractical, because it would greatly increase the costs. Moreover, even when using larger plots, one would still not be certain that the plot is large enough to reach saturation. Here, we propose a more economical alternative. Using the dependence of the basic reproductive number, $R_{0}$, on the plot size in the absence of fungicides, this approach allows to extrapolate a dose-response curve, measured for a particular plot size, to other plot sizes. Figure 5 illustrates possible outcomes. Based on the empirical dose-response curve for the $60 \mathrm{~m}^{2}$ plot and the dependence of $R_{0}$ on the plot size for stripe rust that we determined above in Sec. 3.1 (Fig. 3), we found the dependence of $R_{0}$ on the fungicide dose for different field sizes [Fig. 5(b)]. After that, we computed dose-response curves at different field sizes: an example for a somewhat larger field of $90 \mathrm{~m}^{2}$ is shown as a dashed curve in Fig. 5(a). Thus, the scenario shown in Fig. 5 illustrates how the knowledge of the basic reproductive number can inform strategy of fungicide application. These results should not be considered as quantitatively exact, because we combined the fungicide dose-response data and disease gradient data from different locations. But we believe they illustrate a useful principle and reflect correctly important qualitative trends.

\section{Discussion}

We found that the basic reproductive number, $R_{0}$, of crop pathogens depends on the size and geometry of the field planted with host plants using a single-field, single-season epidemic model. 
$R_{0}$ increases with the field size at small field sizes and saturates to a constant values at large field sizes. The value of $R_{0}$ reaches its maximum in square fields and decreases as the field becomes elongated, while retaining the same area. This is because for smaller and more elongated fields, a larger number of pathogen spores will land outside the field and not reach host plants. This pattern appears to be quite general: it holds for dispersal kernels that decrease exponentially or faster (i.e. Gaussian kernels) as well as for "fat-tailed" dispersal kernels that decrease slower than exponential ones (i.e. power-law kernels). We expect the same qualitative behavior for any dispersal kernel, provided that it is a monotonically decreasing function of the distance $r$ between the source and the target of infection.

As expected, this qualitative picture also holds for the dispersal kernels estimated in wheat stripe rust. The asymptotic values of the basic reproductive number at large field sizes $\left(R_{0 \infty}=35 \pm 3\right.$ for Hermiston 2002, $R_{0 \infty}=23 \pm 4$ for Madras 2002 dataset) are noticeably smaller than the estimate of around 60 that was obtained (Segarra et al., 2001) from the measurements of the apparent rate of infection $r$ (van den Bosch et al., 1988). This difference may result from differences in wheat cultivars, pathogen strains and environmental conditions in these field experiments.

We assumed that the pathogen dispersal is isotropic with the dispersal kernel estimated from the downwind disease gradient. This is a major simplification that will affect the dependence of $R_{0}$ on field size and field geometry. Anisotropy of the dispersal kernels was detected in P. striiformis Soubeyrand et al. (2007) and Mycosphaerella fijiensis Rieux et al. (2014), an important pathogen of banana trees. However, we expect that this assumption does not influence much our estimates of $R_{0 \infty}$. This is because $R_{0 \infty}$ corresponds to the situation when the field is large enough, such that it does not limit pathogen dispersal. Hence, our $R_{0 \infty}$ estimates are determined by spatially-independent estimates of the transmission rate that we obtained from downwind disease gradients. 
The estimates for $R_{0 \infty}$ that we obtained for wheat stripe rust are considerably larger than typical estimates for the basic reproductive number for human or animal diseases. For example, the relatively large values of $R_{0}$ were estimated for childhood diseases such as measles (14-18) and pertussis (5-18) (Anderson and May., 1991), the estimates for the "swine flu" influenza H1N1 were in the range 1.4-1.6 (Fraser et al., 2009), the estimates for rabies were in the range 1-2 (Hampson et al., 2009). A possible exception is malaria, where the estimates of $R_{0}$ between one and more than 3000 were reported (Smith et al., 2007). The $R_{0}$ determines the critical proportion $p_{c}$ of the host population that needs to be immunized in order to eradicate the disease $\left(p_{c}=1-1 / R_{0}\right)$ (Anderson and May., 1991). For example, our estimate for the wheat stripe rust of $R_{0} \simeq 30$ yields the critical proportion $p_{c} \simeq 0.97$. This may be one of the factors to explain why it is so difficult to eradicate rusts, while there are cases of dangerous human diseases (for example, small pox) that were eradicated with the help of vaccination programmes (Anderson and May., 1991). This difference in the values of $R_{0}$ may result from a different biology of hosts (animals versus plants), or, alternatively, it could be due to different nature of the diseases, i. e. systemic diseases in the case of humans and animals versus local lesion diseases in the case of wheat stripe rust. To determine which of these two explanations is more plausible, one needs to estimate $R_{0}$ for systemic disease of plants and local lesion (i. e. skin diseases) of animals. This difference may also be caused by the characteristic features of host populations in agroecosystems, where genetically uniform hosts are planted with high densities in a homogeneous environment. Hence, it would be interesting to compare the $R_{0}$ of crop pathogens with the $R_{0}$ of plant pathogens in natural ecosystems. To make these comparisons valid, one needs to include seasonal cycles of hosts. In the case of annual crops like wheat, this means the consideration of the "between-season" $R_{0}$, in addition to the "within-season" $R_{0}$ discussed in this study.

These findings may help manage some plant diseases, if one knows the spatial scales, i.e. field 
sizes and aspect ratios, over which $R_{0}$ changes considerably. We found that the $R_{0}$ of wheat stripe rust exhibits a large change at a fine spatial scale: when the linear dimension of a square field increases from zero to about thirty meters (Fig. 3). The most substantial change of $R_{0}$ as a function of the field aspect ratio occurs between aspect ratios of 0.01 and 0.2 . These results suggest, that decreasing field sizes and elongating fields may not be a practical measure to control wheat stripe rust, because the beneficial effect of lowering the disease levels is in this case unlikely to outweigh the economical costs associated with using smaller and longer fields. But this method could be feasible for controlling other diseases of crops or pests (for example, western corn rootworm that can disperse over longer distances (Carrasco et al., 2010) than wheat stripe rust). We hope that our study will stimulate more detailed empirical characterization of transmission rates and dispersal kernels for different crop pathogens over long enough distances, such that the framework proposed here could be used to infer how the $R_{0}$ depends on the spatial scales of the host population. Although similar ideas about possibilities to control plant diseases by adjusting field size and geometry were explored mathematically in (Fleming et al., 1982), their framework based on reaction-diffusion models was not capable of including realistic dispersal kernels. Hence, they could not estimate the spatial scales at which the pathogen fitness changes considerably.

The experiments in Hermiston 2002 and Madras 2002 used the same planting density, the same wheat cultivar and the same pathogen race was used for initial inoculation. But the environmental conditions were somewhat different in these two locations. In particular, the inoculation was substantially more successful in Hermiston than in Madras. Hence, we can largely attribute the difference in the disease gradients between these two datasets and the resulting difference in the estimated values of the basic reproductive number to the difference in the environmental conditions. In contrast, in natural epidemics the variation in the outcomes of pathogen dispersal can also result from the genetic variation in pathogen and host population (Tack et al., 2013). 
Therefore, it would be interesting to explore the effect of simulataneously adjusting the spatial scales and introducing genetic diversity to the host population by using host mixtures or multiline cultivars (Mundt, 2002; Mikaberidze et al., 2014a)

From another point of view, our findings could be helpful for choosing the minimum plot sizes and aspect ratios for field experimentation that focuses on transmission properties of plant pathogens. For the experimental plots to be representative of larger fields used by growers in terms of the pathogen's basic reproductive number, the plot size and aspect ratio should be chosen such that they correspond to the start of the saturation of the dependency of $R_{0}$ on the field size (Fig. 3) and aspect ratio (Fig. 4). Thus, our results indicate that in the case of wheat stripe rust, the plot area at which saturation starts is about 0.25 ha and the aspect ratio should be at least 0.2 (this corresponds approximately to a $20 \mathrm{~m} \times 110 \mathrm{~m}$ elongated plot, or, alternatively, a $50 \mathrm{~m} \times 50 \mathrm{~m}$ square plot). In Sec. 3.3 we presented a specific scenario illustrating that the knowledge of the spatial dependence of the basic reproductive number, $R_{0}$, can inform fungicide treatment strategies. Our analysis revealed that in the range of plot sizes typically used to measure the fungicide dose-response curves $\left(20-60 \mathrm{~m}^{2}\right)$, both the disease severity and the basic reproductive number depend strongly on the field size. We proposed a method to extrapolate the dose-response curves measured in small plots to larger plots based on the knowledge of $R_{0}$.

Our results could also help to manage fungicide resistance. Several different fungicides may be applied over smaller, elongated patches within a larger field. In a future study, we plan to determine conditions, when this spatial arrangement of fungicide applications gives the sensitive strain a selective advantage over different resistant strains. This may only work for asexually reproducing pathogens, such as wheat stripe rust outside the Himalayan region and surrounding areas. This strategy allows one to keep the overall field size large enough to be economically advantageous, but requires availability of several different fungicides that have little or no cross-resistance. The same 
reasoning applies also for the case of break-down of disease resistance in host plants. In this case, host cultivars with different disease resistances should be arranged in smaller, elongated patches within a larger field. Favorable arrangements of these patches with different fungicides and host cultivars that would reduce selection for fungicide resistance and minimize break-down of host defences can be investigated using dynamical simulations of the population dynamics model based on Eqs. (1)-(3).

So far we discussed disease control on the level of a single field of crops. But in practice, major crops such as wheat are grown in cultivated landscapes that consist of many fields. Consider the situation when the total area of the landscape and its proportion allocated for wheat cultivation are fixed. Under these constraints, what is an optimal arrangement of wheat plots across the landscape in terms of disease control? Our finding that pathogen fitness decreases in smaller and more elongated fields can be used to optimize the spatial structure of cultivated landscapes in the case when every individual field is far enough from other fields such that inter-field pathogen transmission is negligible. This is only possible when the area allocated for wheat cultivation occupies only a moderate fraction of the total landscape area. But if the area allocated for wheat constitutes a large fraction of the total landscape area, making fields smaller will increase their number and bring them closer to each other. Also, elongated fields may lead to better connectivity between fields in terms of pathogen dispersal. These effects will likely increase the pathogen's basic reproductive number over the landscape scale. We expect that the trade-off between the pathogen transmission within individual fields and between different fields will lead to intermediate optimum field sizes and aspect ratios. To quantify these optima, epidemic models need to include both the scale of single fields and the regional landscape scale (Parnell et al., 2006; Papaïx et al., 2014). Our study lays a solid foundation for future modeling work in this direction. 
482

\section{Acknowledgements}

AM and SB gratefully acknowledge financial support by the ERC advanced grant PBDR 268540

"The population biology of drug resistance: Key principles for a more sustainable use of drugs".

The contributions of CM were supported by NIH grant R01GM96685 through the NSF/NIH

Ecology and Evolution of Infectious Disease Program. The authors would like to thank Kathryn

Sackett for providing estimates of the apparent infection rate, data on disease gradients from replicate plots and helpful discussions. AM is grateful to Bruce McDonald and Roland Regoes for many valuable discussions. AM and SB are grateful to members of the Theoretical Biology group at the ETH Zurich for fruitful discussions and brainstorming.

\section{References}

Anderson, R. M., and R. M. May. 1986. The invasion, persistence and spread of infectious diseases within animal and plant communities. Philosophical transactions of the Royal Society of London. Series B, Biological sciences 314:533-70.

Anderson, R. M., and R. M. May. 1991. Infectious diseases of humans. Oxford University Press.

Bounds, P., J. Blake, B. Fraaije, D. Parsons, S. Knight, F. Burnett, J. Spink, and J. McVittee. 2012. Fungicide performance on winter wheat. Home-Grown Cereals Authority, UK, Project Report 488.

Bowen, K., P. Teng, and A. Roelfs. 1984. Negative Interplot Interference in Field Experiments with Leaf Rust of Wheat. Phytopathology 74:1157-61.

Brown, J. K. M., and M. S. Hovmoller. 2002. Aerial dispersal of pathogens on the global and continental scales and its impact on plant disease. Science (New York, N.Y.) 297:537-41. 
Carrasco, L., T. Harwood, S. Toepfer, A. MacLeod, N. Levay, J. Kiss, R. Baker, J. Mumford, and J. Knight. 2010. Dispersal kernels of the invasive alien western corn rootworm and the effectiveness of buffer zones in eradication programmes in Europe. Annals of Applied Biology 156:63-77.

Cowger, C., L. D. Wallace, and C. C. Mundt. 2005. Velocity of spread of wheat stripe rust epidemics. Phytopathology 95:972-82.

Diekmann, O., J. A. P. Heesterbeek, and J. A. J. Metz. 1990. On the definition and the computation of the basic reproduction ratio $\mathrm{R} 0$ in models for infectious diseases in heterogeneous populations. Journal of Mathematical Biology 28:365-382.

Filipe, J. A. N., R. C. Cobb, R. K. Meentemeyer, C. A. Lee, Y. S. Valachovic, A. R. Cook, D. M. Rizzo, and C. A. Gilligan. 2012. Landscape epidemiology and control of pathogens with cryptic and long-distance dispersal: sudden oak death in northern Californian forests. PLoS computational biology 8:e1002328.

Fitt, B. D. L., P. H. Gregory, A. D. Todd, H. A. McCartney, and O. C. Macdonald. 1987. Spore Dispersal and Plant Disease Gradients; a Comparison between two Empirical Models. Journal of Phytopathology 118:227-242.

Fleming, R., L. Marsh, and H. Tuckwell. 1982. Effect of field geometry on the spread of crop disease. Protection Ecology 4:81.

Frantzen, J., and F. V. D. Bosch. 2000. Spread of organisms: can travelling and dispersive waves be distinguished ? Basic and Applied Ecology 91:83-91.

Fraser, C., C. A. Donnelly, S. Cauchemez, W. P. Hanage, M. D. Van Kerkhove, T. D. Hollingsworth, J. Griffin, R. F. Baggaley, H. E. Jenkins, E. J. Lyons, T. Jombart, W. R. Hinsley, 
N. C. Grassly, F. Balloux, A. C. Ghani, N. M. Ferguson, A. Rambaut, O. G. Pybus, H. Lopez-Gatell, C. M. Alpuche-Aranda, I. B. Chapela, E. P. Zavala, D. M. E. Guevara, F. Checchi, E. Garcia, S. Hugonnet, and C. Roth. 2009. Pandemic potential of a strain of influenza A (H1N1): early findings. Science (New York, N.Y.) 324:1557-61.

Gibson, G. J., W. Otten, J. A. N. Filipe, A. Cook, G. Marion, and C. A. Gilligan. 2006. Bayesian estimation for percolation models of disease spread in plant populations. Statistics and Computing 16:391-402.

Gubbins, S., C. A. Gilligan, and A. Kleczkowski. 2000. Population dynamics of plant-parasite interactions: thresholds for invasion. Theoretical Population Biology 57:219-33.

Hall, R. J., S. Gubbins, and C. A. Gilligan. 2007. Evaluating the performance of chemical control in the presence of resistant pathogens. Bulletin of mathematical biology 69:525-37.

Hampson, K., J. Dushoff, S. Cleaveland, D. T. Haydon, M. Kaare, C. Packer, and A. Dobson. 2009. Transmission dynamics and prospects for the elimination of canine rabies. PLoS Biology 7:e53.

Heesterbeek, J. 2002. A Brief History of R 0 and a Recipe for its Calculation. Acta Biotheoretica 5:189-204.

Keeling, M. J., and P. Rohani, 2008. Spatial Models. Chapter 7, page 266 in Modeling Infectious Diseases in Humans and Animals. Princeton University Press, Princeton.

Long, D., 2003. Estimated small grain losses from rust in 2002 http://www.ars.usda.gov/SP2UserFiles/ad_hoc/36400500Smallgrainlossesduetorust/2002loss/02rustloss.pdf.

Mazzi, D., and S. Dorn. 2012. Movement of insect pests in agricultural landscapes. Annals of Applied Biology 160:97-113. 
McDonald, B. A., and C. Linde. 2002. Pathogen population genetics, evolutionary potential, and durable resistance. Annual Review of Phytopathology 40:349-79.

Medlock, J., and M. Kot. 2003. Spreading disease: integro-differential equations old and new. Mathematical Biosciences 184:201-222.

Mikaberidze, A., B. McDonald, and S. Bonhoeffer. 2014a. Developing smarter host mixtures to control plant disease. Plant Pathology in press DOI:0.1111/ppa.12321, e-print arXiv:1402.2788 .

Mikaberidze, A., B. A. McDonald, and S. Bonhoeffer. 2014b. Can high risk fungicides be used in mixtures without selecting for fungicide resistance? Phytopathology 104:324-331.

Mollison, D. 1977. Spatial Contact Models for Ecological and Epidemic Spread. Journal of the Royal Statistical Society. Series B 39:283.

Montarry, J., F. M. Hamelin, I. Glais, R. Corbi, and D. Andrivon. 2010. Fitness costs associated with unnecessary virulence factors and life history traits: evolutionary insights from the potato late blight pathogen Phytophthora infestans. BMC evolutionary biology 10:283.

Mundt, C. C. 2002. Use of multiline cultivars and cultivar mixtures for disease management. Annual review of phytopathology 40:381-410.

Mundt, C. C. 2014. Durable resistance: A key to sustainable management of pathogens and pests. Infection, genetics and evolution: journal of molecular epidemiology and evolutionary genetics in infectious diseases in press.

Mundt, C. C., L. S. Brophy, and S. C. Kolar. 1996. Effect of genotype unit number and spatial arrangement on severity of yellow rust in wheat cultivar mixtures. Plant Pathology 45:215-222. 
Nathan, R., E. Klein, J. J. Robledo-Arnuncio, and E. Revilla, 2012. Dispersal kernels: review.

Chapter 15, page 187 in J. Clobert, M. Baguette, T. G. Benton, and J. M. Bullock, editors.

Dispersal ecology and evolution. Oxford Univ. Press.

Papaïx, J., S. Touzeau, H. Monod, and C. Lannou. 2014. Can epidemic control be achieved by altering landscape connectivity in agricultural systems? Ecological Modelling 284:35-47.

Park, A. W., S. Gubbins, and C. A. Gilligan. 2001. Invasion and persistence of plant parasites in a spatially structured host population. Oikos 94:162-174.

Parnell, S., C. Gilligan, and F. van den Bosch. 2005. Small-scale fungicide spray heterogeneity and the coexistence of resistant and sensitive pathogen strains. Phytopathology 95:632-9.

Parnell, S., F. van den Bosch, and C. Gilligan. 2006. Large-scale fungicide spray heterogeneity and the regional spread of resistant pathogen strains. Phytopathology 96:549-55.

Paveley, N., M. Hims, and D. Stevens. 1998. Appropriate fungicide doses for winter wheat and matching crop management to growth and yield potential. Home-Grown Cereals Authority, UK, Project Report 166.

Paysour, R., and W. Fry. 1983. Interplot interference: A model for planning field experiments with aerially disseminated pathogens. Phytopathology 73:1014.

Rieux, A., S. Soubeyrand, F. Bonnot, E. K. Klein, J. E. Ngando, A. Mehl, V. Ravigne, J. Carlier, and L. De Lapeyre De Bellaire. 2014. Long-distance wind-dispersal of spores in a fungal plant pathogen: Estimation of anisotropic dispersal kernels from an extensive field experiment. PLoS ONE 9.

Sache, I., and C. Vallavieille-Pope. 1993. Comparison of the wheat brown and yellow rusts for 
monocyclic sporulation and infection processes, and their polycyclic consequences. Journal of phytopathology 138:55-65.

Sackett, K. E., and C. C. Mundt. 2005a. Primary disease gradients of wheat stripe rust in large field plots. Phytopathology 95:983-91.

Sackett, K. E., and C. C. Mundt. 2005b. The effects of dispersal gradient and pathogen life cycle components on epidemic velocity in computer simulations. Phytopathology 95:992-1000.

Sackett, K. E., and C. C. Mundt. 2009. Effect of plot geometry on epidemic velocity of wheat yellow rust. Plant Pathology 58:370-377.

Segarra, J., M. J. Jeger, and F. van den Bosch. 2001. Epidemic dynamics and patterns of plant diseases. Phytopathology 91:1001-10.

Shaw, M. W., and M. Pautasso. 2014. Networks and Plant Disease Management: Concepts and Applications. Annual Review of Phytopathology 52:in press.

Smith, D. L., F. E. McKenzie, R. W. Snow, and S. I. Hay. 2007. Revisiting the basic reproductive number for malaria and its implications for malaria control. PLoS Biology 5 :e42.

Soubeyrand, S., J. Enjalbert, A. Sanchez, and I. Sache. 2007. Anisotropy, in density and in distance, of the dispersal of yellow rust of wheat: experiments in large field plots and estimation. Phytopathology 97:1315-24.

Strange, R. N., and P. R. Scott. 2005. Plant disease: a threat to global food security. Annual Review of Phytopathology 43:83-116.

Strengbom, J., G. Englund, and L. Ericson. 2006. Experimental scale and precipitation modify effects of nitrogen addition on a plant pathogen. Journal of Ecology 94:227-233. 
Tack, A., J. Hakala, T. Petäjä, M. Kulmala, and A. Laine. 2013. Genotype and spatial structure shape pathogen dispersal and disease dynamics at small spatial scales. Ecology 95:703-714.

van den Berg, F., N. Bacaer, J. S. J. Metz, C. Lannou, and F. van den Bosch. 2011. Periodic host absence can select for higher or lower parasite transmission rates. Evolutionary Ecology 25:121-137.

van den Bosch, F., H. Frinking, J. Metz, and J. Zadoks. 1988. Focus expansion in plant disease. III: Two experimental examples. Phytopathology 78:919-925.

van den Bosch, F., and C. A. Gilligan. 2008. Models of fungicide resistance dynamics. Annu. Rev. Phytopathol. 46:123-47.

van den Bosch, F., N. McRoberts, F. van den Berg, and L. Madden. 2008. The Basic Reproduction Number of Plant Pathogens: Matrix Approaches to Complex Dynamics. Phytopathology 98:239-249.

Vanderplank, J. E. 1963. Plant Diseases: Epidemics and Control. Academic Press, New York. Wellings, C. R. 2011. Global status of stripe rust: a review of historical and current threats. Euphytica 179:129-141.

\section{Supplemental Material}

\section{Ecological Archives}

Appendix A "Mathematical methods and estimation techniques for determination of the basic reproductive number" is available online: 
Table 1: Variables and parameters

Description

Dimension

\begin{tabular}{|c|c|c|}
\hline \multicolumn{3}{|l|}{ Variables } \\
\hline$H(x, y, t)$ & Density of healthy host tissue & $\mathrm{dl}$ \\
\hline$I(x, y, t)$ & Density of infected host tissue & $\mathrm{dl}$ \\
\hline \multicolumn{3}{|l|}{ Parameters } \\
\hline$d_{x}, d_{y}$ & Linear dimensions of the field along $x$ and $y$ & $\mathrm{~m}$ \\
\hline$a$ & Characteristic spatial scale of pathogen dispersal (dispersal radius) & $\mathrm{m}$ \\
\hline$\beta$ & Transmission rate & days $^{-1}$ \\
\hline$\mu^{-1}$ & Average infectious period & days \\
\hline$r_{H}$ & Growth rate of healthy host tissue & days $^{-1}$ \\
\hline$K$ & "Carrying capacity" of the healthy host tissue & $\mathrm{dl}$ \\
\hline$R_{0 \infty}$ & Basic reproductive number in the limit of a very large field & $\mathrm{dl}$ \\
\hline \multicolumn{3}{|l|}{ Functions } \\
\hline$\kappa(r)$ & Dispersal kernel & $\mathrm{m}^{-1}$ \\
\hline$R_{0}\left(d_{x}, d_{y}\right)$ & Basic reproductive number & $\mathrm{dl}$ \\
\hline$\lambda(x, y)$ & The force of infection [Eq. (4)] & \\
\hline
\end{tabular}


Figure 1. Basic reproductive number $R_{0}$ as a function of the field size $d$ for the two-dimensional field according to the numerical solution of Eq. (6) (solid green) using (i) the Gaussian [Eq. (A.21) at $n=2, a=10 \mathrm{~m}$ ], (ii) the exponential [Eq. (A.21) at $n=1, a=10 \mathrm{~m}$ ] and (iii) the power law dispersal kernel [Eq. (A.19) at $r_{0}=1 \mathrm{~m}, b=2.1$ ]. Model parameters: $R_{0 \infty}=\beta K / \mu=2$.

Figure 2. Disease severity of wheat stripe rust is plotted as a function of the distance from focus, according to outcomes of field experiments (Sackett and Mundt, 2005a; Cowger et al., 2005). Two datasets, Hermiston 2002 downwind (left panel) and Madras 2002 downwind were fitted with the exponential function [Eq. (A.21) with $n=1$, dashed curve], the Gaussian function [Eq. (A.21) with $n=2$, dotted curve] and the modified power-law function [Eq. (A.19), solid curve].

Figure 3. Basic reproductive number $R_{0}$ as a function of the field size $d$ of a square field calculated [by solving numerically the eigenvalue problen Eq. (6)] using the modified power-law dispersal kernel [Eq. (10)] fitted in Fig. 2 to disease gradient datasets (i) Hermiston 2002 downwind (solid curve), and (ii) Madras 2002 downwind (dashed curve) obtained in (Sackett and Mundt, 2005a; Cowger et al., 2005). Horizontal dashed lines show the asymptotic values of the basic reproductive number at large field sizes, $R_{0 \infty}$, for Hermiston 2002 (upper line) and Madras 2002 (lower line) datasets. Error bars represent $95 \%$ confidence intervals for $R_{0 \infty}$ estimates (see Appendix A.3).

Figure 4. Basic reproductive number $R_{0}$ as a function of the field aspect ratio $d_{x} / d_{y}$ (the field area $S=d_{x} d_{y}$ was kept the same). The calculation was performed numerically using the power-law dispersal kernels fitted to disease gradient data (Fig. 2) from Hermiston 2002 (upper panel) and Madras 2002 (lower panel) datasets obtained in (Sackett and Mundt, 2005a; Cowger et al., 2005). Different curves show the $R_{0}$ for different field areas: $S=4$ ha (yellow solid), $S=1$ ha (blue dashed), $S=0.37$ ha (red dash-dotted), $S=0.04$ ha (orange dotted). 
649 Figure 5. Dependence of stripe rust severity (panel (a)) and the basic reproductive number, $R_{0}$, 650 (panel (b)) on the fungicide dose (epoxiconazole) for two different sizes of square fields:

$651 \quad S=60 \mathrm{~m}^{2}$ (blue, solid) and $S=90 \mathrm{~m}^{2}$ (red, dashed). 


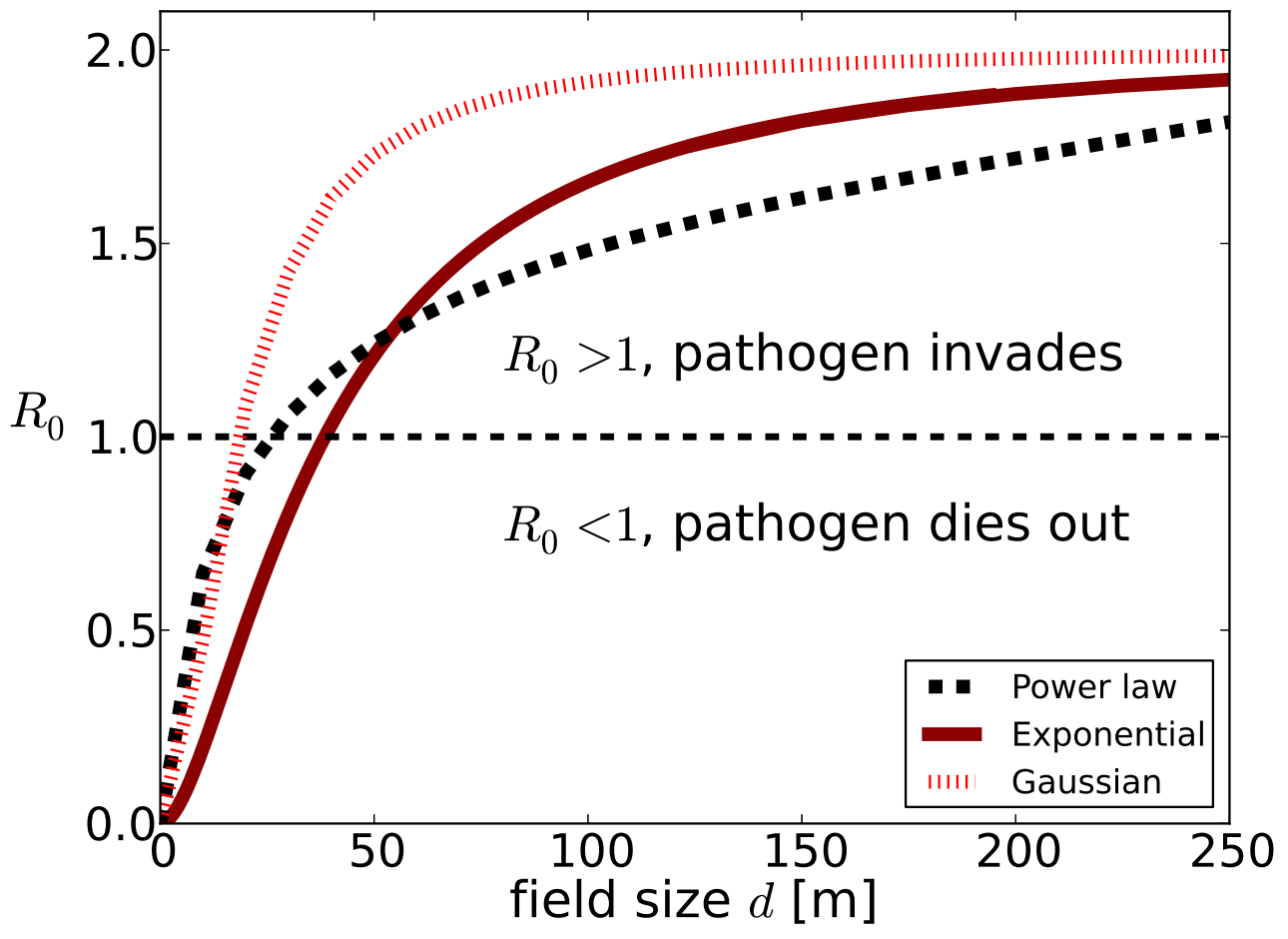

Figure 1 
A. Mikaberidze et al.

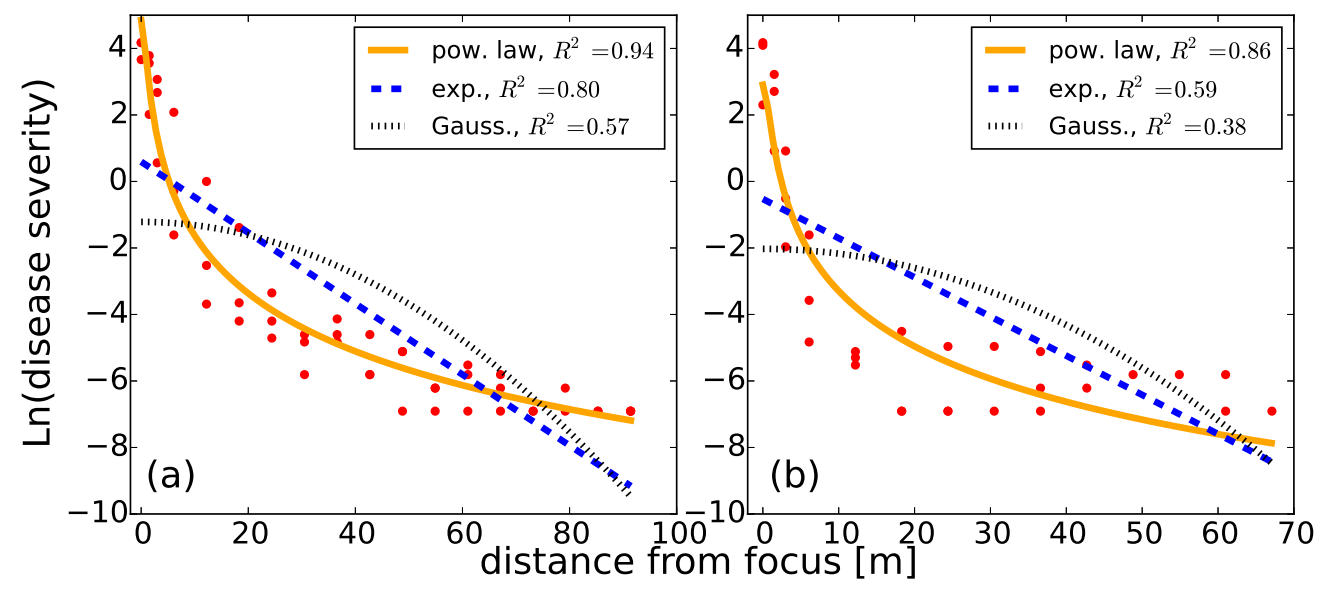

Figure 2 
A. Mikaberidze et al.

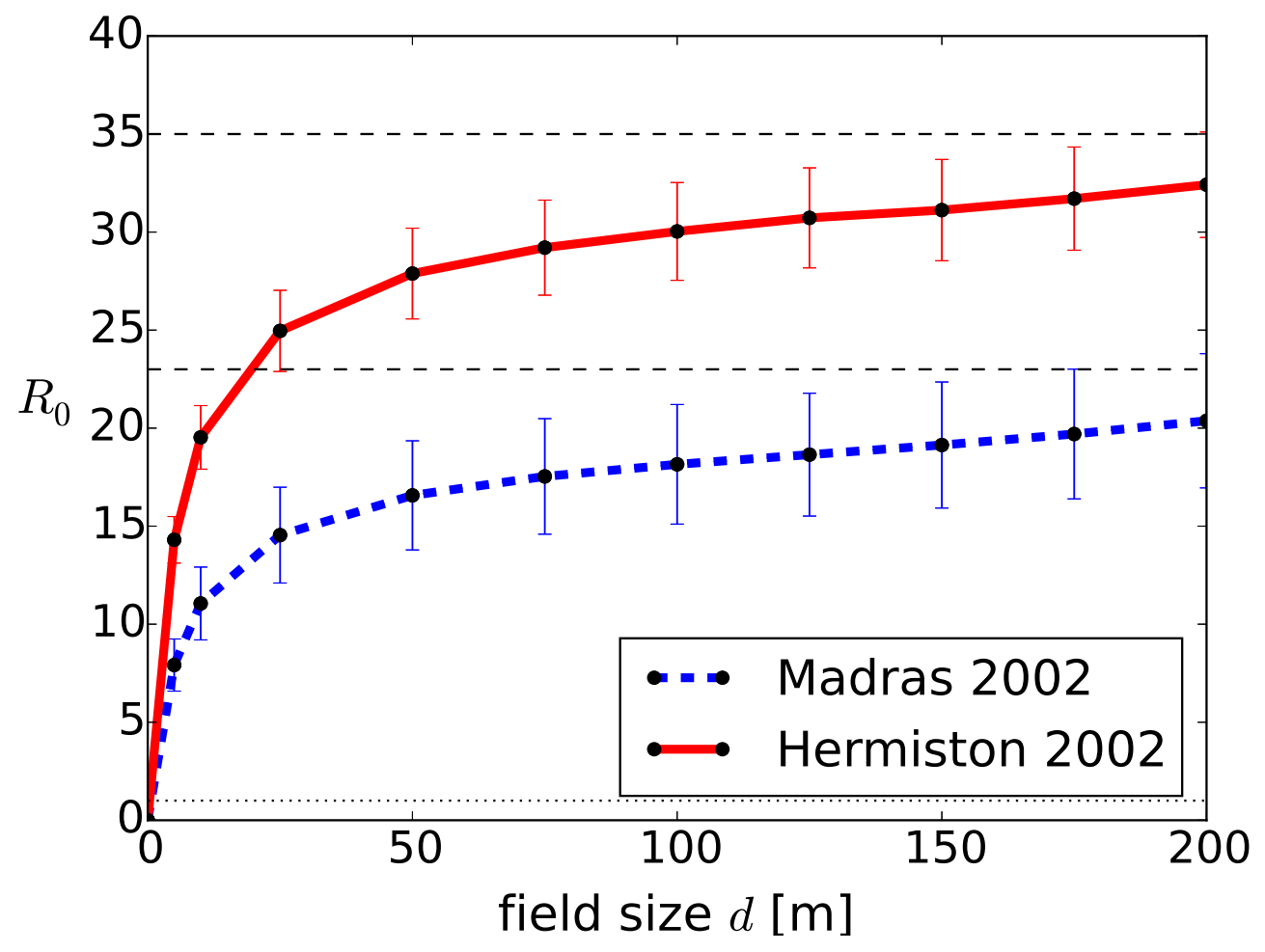

Figure 3 
A. Mikaberidze et al.

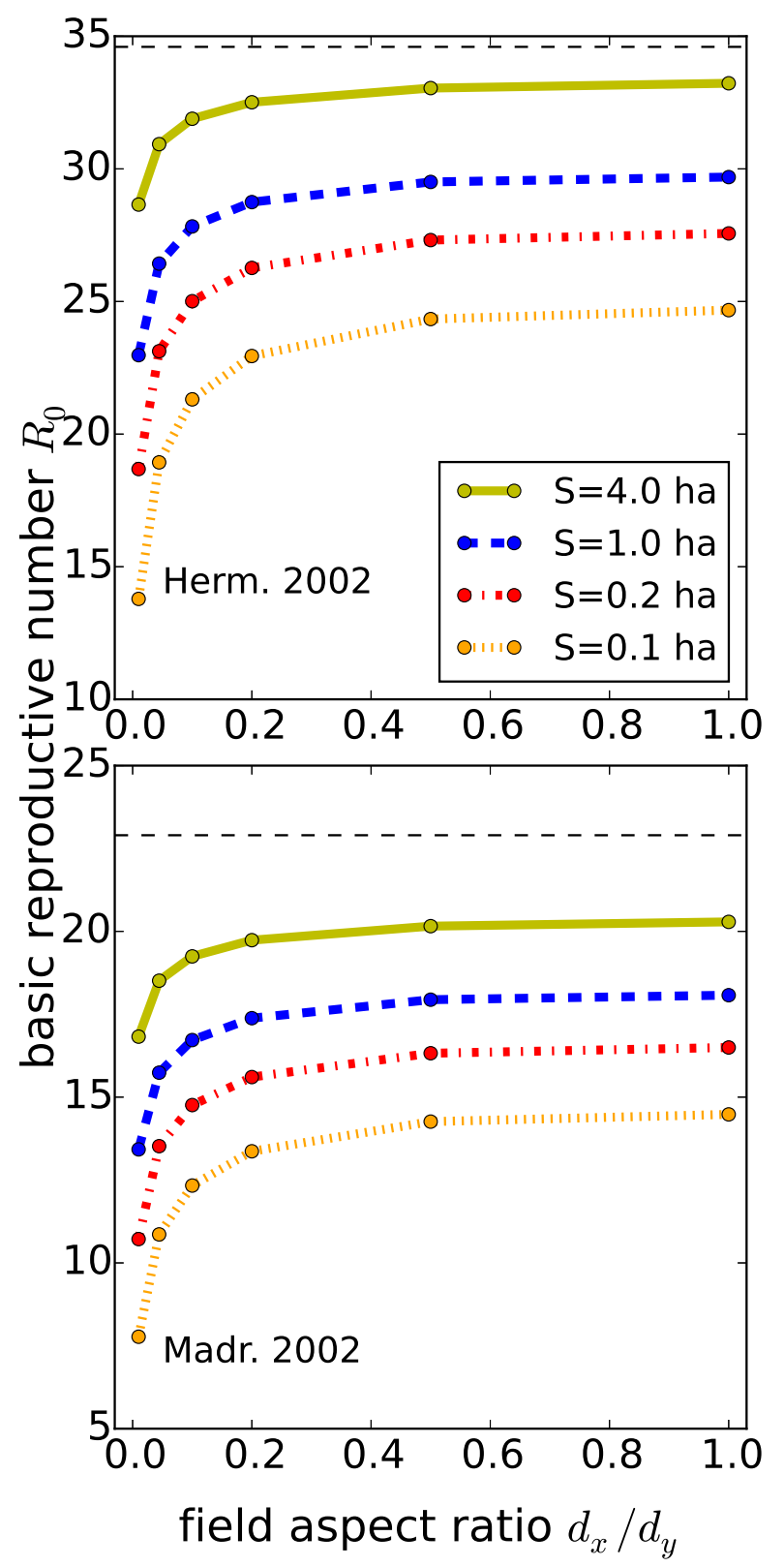

Figure 4 
A. Mikaberidze et al.
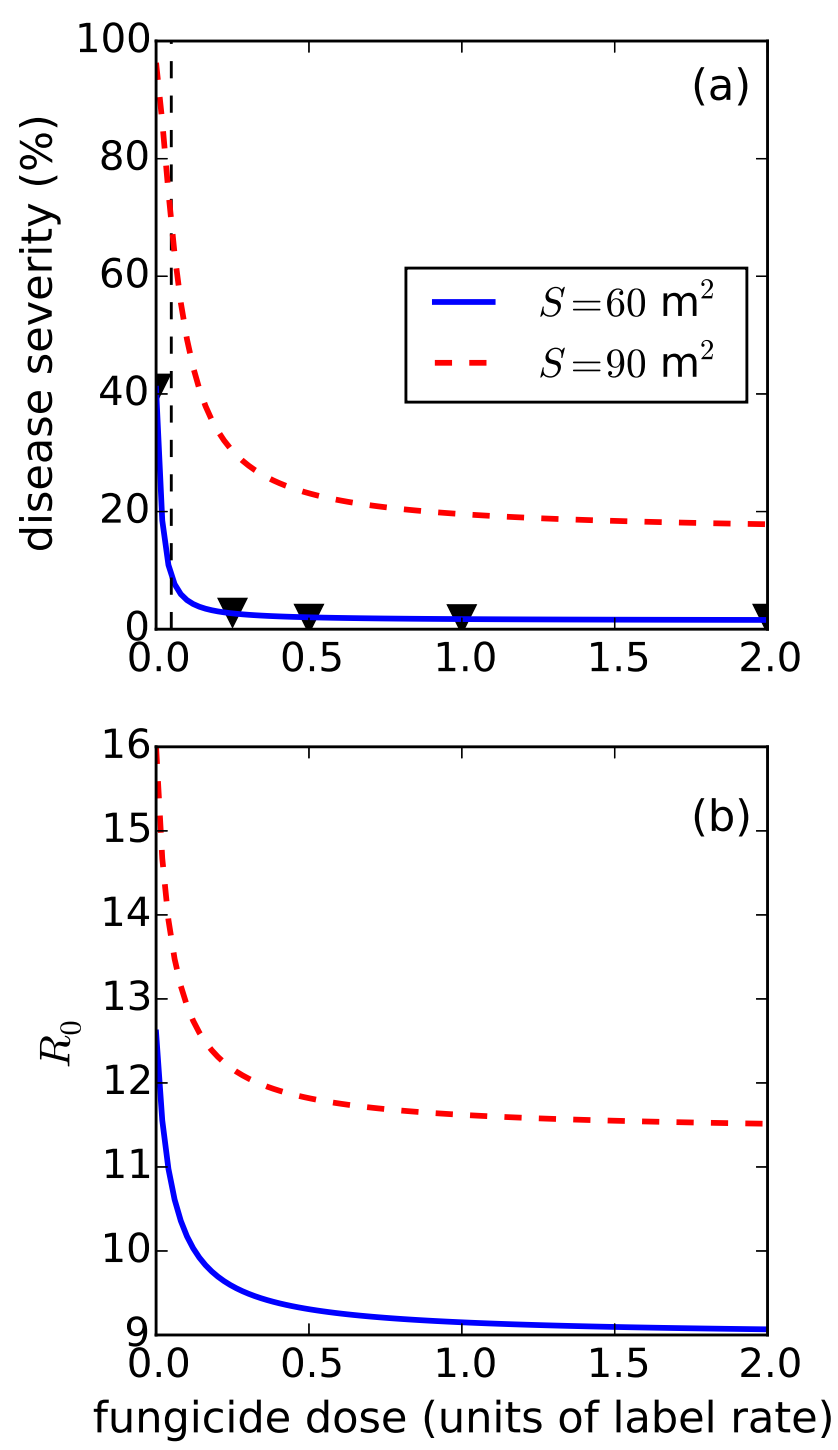

Figure 5 